\title{
The influence of ribosome modulation factor on the survival of stationary-phase Escherichia coli during acid stress
}

\author{
Walid M. El-Sharoudt and Gordon W. Niven \\ Food Microbial Sciences Unit, School of Food Biosciences, The University of Reading, Reading \\ RG6 6AP, UK
}

Correspondence

Walid M. El-Sharoud

wmel_sharoud@mans.edu.eg

Received 19 August 2006

Revised 26 September 2006

Accepted 3 October 2006

\begin{abstract}
Ribosome modulation factor (RMF) was shown to have an influence on the survival of Escherichia coli under acid stress during stationary phase, since the viability of cultures of a mutant strain lacking functional RMF decreased more rapidly than that of the parent strain at $\mathrm{pH} 3$. Loss of ribosomes was observed in both strains when exposed to low $\mathrm{pH}$, although this occurred at a higher rate in the RMF-deficient mutant strain, which also suffered from higher levels of rRNA degradation. It was concluded that the action of RMF in limiting the damage to rRNA contributed to the protection of $E$. coli under acid stress. Expression of the $r m f$ gene was lower during stationary phase after growth in acidified media compared to media containing no added acid, and the increased $r m f$ expression associated with transition from exponential phase to stationary phase was much reduced in acidified media. It was demonstrated that RMF was not involved in the stationary-phase acid-tolerance response in $E$. coli by which growth under acidic conditions confers protection against subsequent acid shock. This response was sufficient to overcome the increased vulnerability of the RMF-deficient mutant strain to acid stress at $\mathrm{pH}$ values between 6.5 and 5.5.
\end{abstract}

\section{INTRODUCTION}

Ribosome modulation factor (RMF) is a ribosomeassociated protein that is synthesized in Escherichia coli on entry into stationary phase (Wada et al., 1990; Yamagishi et al., 1993) and during slow growth (Yamagishi et al., 1993; Izutsu et al., 2001; El-Sharoud \& Niven, 2005). Mutant strains lacking functional RMF show reduced survival during stationary phase (Yamagishi et al., 1993; Apirakaramwong et al., 1998) and are also more vulnerable to osmotic stress (Garay-Arroyo et al., 2000) and heat stress (Niven, 2004) during stationary phase. It is therefore generally considered that RMF is a protective factor of ribosomes during times of slow growth. The synthesis of RMF by the cell is associated with the detection of $100 \mathrm{~S}$ ribosome dimers in cell-free extracts, and it has been suggested that such dimers represent an inactive stabilized 'storage form' of ribosomes (Fukuchi et al., 1995; Wada, 1998; Ishihama, 1999). During heating of stationary-phase cultures, $100 \mathrm{~S}$ particles were shown to dissociate more rapidly than $70 \mathrm{~S}$ ribosomes, indicating that they are not inherently more heat stable (Niven, 2004). It was therefore not possible to attribute increased ribosome stability directly to dimerization in this case. Since they have not yet been

tPresent address: Faculty of Agriculture, Mansoura University, Mansoura, Egypt.

Abbreviations: ATR, acid-tolerance response; RMF, ribosome modulation factor. observed in intact cells, the exact nature of the ribosomal dimers and the contribution they make to RMF function are therefore currently uncertain.

In a previous study of the influence of RMF on cells during acid stress in exponential phase, we demonstrated increased expression of the $r m f$ gene that was inversely proportional to growth rate under acidic conditions (El-Sharoud \& Niven, 2005). This is consistent with growth rate control of rmf expression mediated by ppGpp, as described by Izutsu et al. (2001). However, increased rmf expression during exponential phase did not result in ribosome dimerization, and no difference in acid resistance was observed between a mutant strain lacking functional RMF and the parent strain under these conditions. It was not possible, therefore, to correlate RMF production, ribosome dimerization and acid stress resistance during exponential-phase growth. As peptide chain elongation rate was higher in the parent strain than the mutant strain during acid stress in exponential phase, it was speculated that the function of RMF under such conditions may be to facilitate more efficient protein synthesis. This may be achieved by inactivating excess ribosomes to maintain the required balance between the concentrations of ribosomes and protein synthesis factors and therefore reduce ribosome stalling (El-Sharoud \& Niven, 2005). This idea is consistent with the more accepted idea of RMF mediating the formation of a storage ribosome form in stationary phase, since both involve ribosome inactivation. 
It appears that the importance of RMF to stress resistance depends on both the physiological state of the cell and the nature of the environmental stress. It is also clear that the exact function of RMF and the means by which this is achieved are not yet fully understood. In order to further examine these issues in relation to acid stress, and to complement our previous studies of exponential-phase cultures, we have carried out a study of the influence of RMF on stationary-phase $E$. coli cultures under acidic conditions.

\section{METHODS}

Cultures and growth conditions. Escherichia coli W3110 and two mutant strains derived from it, HMY15 and HMY13, were the generous gift of Professor Akira Ishihama, National Institute of Genetics, Shizouka, Japan. The $r m f$ gene of strain HMY15 is disrupted by insertion of a chloramphenicol-resistance gene (Yamagishi et al., 1993), and E. coli HMY13 carries an in-frame translational rmf-lacZ gene fusion and a chloramphenicol-resistance gene (Yamagishi et al., 1993). Strain W3110 was maintained on tryptone soya agar (TSA) (Oxoid), supplemented with chloramphenicol $\left(10 \mu \mathrm{g} \mathrm{ml}^{-1}\right)$ in the case of E. coli HMY15 and HMY13. All cultures were grown in tryptone soya broth (TSB) (Oxoid) at $37^{\circ} \mathrm{C}$ with shaking. Stationary-phase cultures for experimentation were obtained by inoculating $10 \mathrm{ml}$ medium with a single colony and incubating for $24 \mathrm{~h}$. This was subcultured into fresh medium $(1 \%$ $\mathrm{v} / \mathrm{v}$ ) and incubated for a further $18 \mathrm{~h}$. The $\mathrm{pH}$ of TSB prior to inoculation was $7.3 \pm 0.2$. When required, media $\mathrm{pH}$ values were adjusted by the addition of $\mathrm{HCl}$. Viable counts were made by plating on TSA following serial dilution in maximum recovery diluent (Oxoid) and incubation at $37^{\circ} \mathrm{C}$ for $24 \mathrm{~h}$.

Ribosome analysis by sucrose density-gradient centrifugation. Ribosome analysis was carried out using the sucrose densitygradient centrifugation technique described by Maki et al. (2000) and Izutsu et al. (2001). Samples of cultures were centrifuged at $10000 \mathrm{~g}$ for $15 \mathrm{~min}$ at $4{ }^{\circ} \mathrm{C}$, and the pellet was resuspended in buffer containing $10 \mathrm{mM}$ Tris/ $\mathrm{HCl}, 10 \mathrm{mM}$ magnesium acetate and $100 \mathrm{mM}$ ammonium acetate (pH 7.6) and vortexed with acidwashed glass beads $\left(0.3 \mathrm{~mm}\right.$ diameter, Sigma) for $5 \mathrm{~min}$ at $4{ }^{\circ} \mathrm{C}$. The resultant suspension was centrifuged at $15000 \mathrm{~g}$ for $10 \mathrm{~min}$ at $4{ }^{\circ} \mathrm{C}$. The supernatant was kept on ice and the pellet was resuspended in the above buffer, vortexed and centrifuged as before. This was repeated twice. Samples of the composite supernatant were loaded onto 5-20\% sucrose gradients and centrifuged at 25000 r.p.m. for $3 \mathrm{~h}$ at $5{ }^{\circ} \mathrm{C}$ in a Beckman SW 40Ti rotor (maximum $\mathrm{RCF}=75000 \mathrm{~g}$ ) using a Beckman L8-55M ultracentrifuge (Beckman Instruments). Sucrose gradients were analysed by upward displacement with $50 \%$ sucrose solution with continuous monitoring of absorbance at $254 \mathrm{~nm}$. Data were normalized to absorbance at $280 \mathrm{~nm}$.

RNA analysis using agarose gel electrophoresis. RNA analyses were carried out according to the method of Kornblum et al. (1988) using cell lysates of E. coli cultures grown in TSB. Culture volumes containing approximately $10^{8}$ c.f.u. $\mathrm{ml}^{-1}$ were centrifuged at $10000 \mathrm{~g}$ for $15 \mathrm{~min}$ at $4{ }^{\circ} \mathrm{C}$. The pellet was resuspended in $100 \mu \mathrm{l}$ lysis buffer ( $\mathrm{pH} 7.6$ ) containing $20 \%$ sucrose, $20 \mathrm{mM}$ Tris/ $\mathrm{HCl}$, $10 \mathrm{mM}$ EDTA, $50 \mathrm{mM} \mathrm{NaCl}$ and $1 \mathrm{mg}$ lysozyme $\mathrm{ml}^{-1}$. The suspension was incubated on ice until it became viscous (approx. $15 \mathrm{~min}$ ). SDS $\left(100 \mu \mathrm{l}\right.$ of $2 \%$ solution) and proteinase $\mathrm{K}\left(10 \mu \mathrm{l}\right.$ of $5 \mathrm{mg} \mathrm{ml}^{-1}$ solution) were then added and the mixture was shaken at room temperature for $15 \mathrm{~min}$. The lysate was twice frozen at $-70{ }^{\circ} \mathrm{C}$ and thawed at $45^{\circ} \mathrm{C}$. It was then mixed with $50 \mu \mathrm{l}$ loading buffer containing $50 \%$, v/v, glycerol, $200 \mathrm{mM}$ EDTA, $0.1 \%$ bromophenol blue and $0.1 \%$ xylene cyanol dissolved in running buffer. Samples $(10 \mu \mathrm{l})$ were loaded onto $1.2 \%$ agarose gels containing $1 \mu \mathrm{g}$ ethidium bromide $\mathrm{ml}^{-1}$. Running buffer ( $\mathrm{pH} 7.0$ ) contained $20 \mathrm{mM}$ MOPS, $5 \mathrm{mM}$ sodium acetate and $0.1 \mathrm{mM}$ EDTA (Sigma). Electrophoresis was carried out at $80 \mathrm{~V}$.

Assay of $\boldsymbol{\beta}$-galactosidase activity. Expression of $r m f$ was estimated using E. coli strain HMY13, which expresses $\beta$-galactosidase from the rmf promoter. The enzyme was assayed in toluene-permeabilized cells (Miller, 1972) as follows. Samples of culture $(20 \mu \mathrm{l})$ were added to $0.8 \mathrm{ml}$ buffer containing $10 \mathrm{mM}$ Tris/ $\mathrm{HCl}, 10 \mathrm{mM}$ $\mathrm{KCl}$ and $1 \mathrm{mM} \mathrm{MgSO}_{4}, \mathrm{pH}$ 7.0. Toluene $(40 \mu \mathrm{l})$ was added and samples were incubated at $37^{\circ} \mathrm{C}$ for $10 \mathrm{~min}$. The enzymic reaction was initiated by the addition of $0.2 \mathrm{ml} 25 \mathrm{mM} o$-nitrophenyl $\beta$-Dgalactopyranoside and incubation at $37^{\circ} \mathrm{C}$. After incubation to an absorbance at $420 \mathrm{~nm}$ of $0.2-0.4$, the reaction was terminated by the addition of $0.4 \mathrm{ml} 1 \mathrm{M} \mathrm{Na}_{2} \mathrm{CO}_{3}$. The samples were then centrifuged at $10000 \mathrm{~g}$ for $10 \mathrm{~min}$ and the absorbance of the supernatant was measured at $420 \mathrm{~nm}$. The concentration of product was determined using a molar absorption coefficient of $4500 \mathrm{M}^{-1} \mathrm{~cm}^{-1}$, and one unit of enzyme activity was defined as the amount required to form $1 \mu \mathrm{mol}$ product $\mathrm{min}^{-1}$. Activities were normalized to the optical density of the culture at $600 \mathrm{~nm}$.

\section{RESULTS}

\section{Effect of RMF on the survival of $E$. coli at pH 3}

The influence of RMF on the survival of stationary-phase E. coli cultures under acid stress was investigated by comparing the viability of an RMF-deficient mutant strain (HMY15) with that of the parent strain (W3110) after inoculation into TSB medium acidified to $\mathrm{pH}$ 3. These strains of $E$. coli were shown to be incapable of growth at $\mathrm{pH}$ levels lower than $\mathrm{pH} 4$ (data not shown). Over a period of $5 \mathrm{~h}$, there was a relatively small decrease in the viable count of the parent strain of approximately $0.8 \log$ units (Fig. 1). The mutant strain was found to be more vulnerable to acid stress than the parent strain, the viable count of strain HMY15 having decreased by approximately $3 \log$ units after

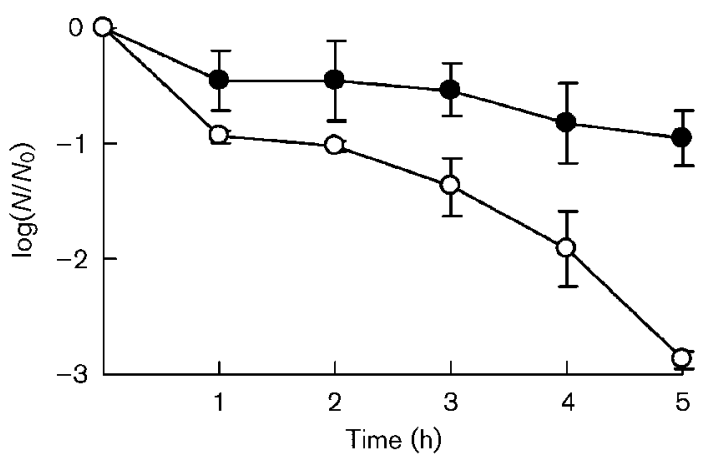

Fig. 1. Viability of E. coli W3110 (O) and the RMF-deficient mutant strain HMY15 $(\bigcirc)$ following the inoculation of stationary-phase culture into fresh medium adjusted to $\mathrm{pH} 3$. The viable counts were approximately $2 \times 10^{7}$ c.f.u. $\mathrm{ml}^{-1}$ immediately after addition to the acidified medium. Values presented are the means of three separate experiments and the error bars represent \pm 1 SD. 
$5 \mathrm{~h}$. These observations suggested that a lack of functional RMF was detrimental to the survival of stationary-phase cultures of $E$. coli under acid stress.

\section{Relative ribosome particle composition of $E$. coli strains during acid stress}

The influence of acid stress on relative amounts of ribosome particles was investigated by sucrose density-gradient centrifugation of cell-free extracts of the mutant and parent strains after exposure to $\mathrm{pH}$ 3, adjusted by $\mathrm{HCl}$. As shown in Fig. 2, a large proportion of the ribosomes in the stationary-phase inoculum of strain W3110 was present as 100S dimers, although a large number of $70 \mathrm{~S}$ particles was also observed. Extracts of strain HMY15 contained no dimers and most of the ribosomes were present as $70 \mathrm{~S}$ particles, consistent with similar analyses reported elsewhere (Wada et al., 1990; Yamagishi et al., 1993). When cultures were analysed immediately after exposure to $\mathrm{pH} 3$ (within the $20 \mathrm{~min}$ taken to harvest and disrupt the cells), $100 \mathrm{~S}$ dimers were no longer detected in extracts of the parent strain, and only $30 \mathrm{~S}, 50 \mathrm{~S}$ and $70 \mathrm{~S}$ particles were observed. Within the same time scale, most of the $70 \mathrm{~S}$ ribosomes in the mutant strain had dissociated into ribosome subunits. After $5 \mathrm{~h}$, no intact ribosome subunits were observed in the mutant strain and only a relatively small amount of 50S subunits were detected in the parent strain. From these data, it is clear that extensive ribosome particle dissociation took place in stationary-phase cultures of both strains during acid stress. Although this was more rapid in the RMF-deficient strain, there was no evidence that the ability to synthesize RMF resulted in the retention of significant intact ribosomes in stressed cultures of the parent strain.

To further explore the influence of RMF on ribosome integrity under acid stress, analyses of rRNA were carried out after acidification of stationary-phase cultures of both parent and mutant strains to $\mathrm{pH}$ 3. In each case, two bands were detected on agarose gels that were shown by comparison with known standards to represent the $16 \mathrm{~S}$ and $23 \mathrm{~S}$ rRNA species (Fig. 3). The level of rRNA degradation was found to be visibly greater in the mutant strain than in the parent strain after exposure to acid stress. This can be inferred from the reduction in the intensity of the bands and increased smearing in the gels. It therefore appeared that the role of RMF in the survival of E. coli under acid stress during stationary phase may be associated more with the preservation of rRNA than with the maintenance of ribosome subunit structure.

\section{Influence of growth pH on rmf expression and ribosome dimerization during stationary phase}

In the above experiments, cells were grown to stationary phase in unacidified medium before being exposed to acid
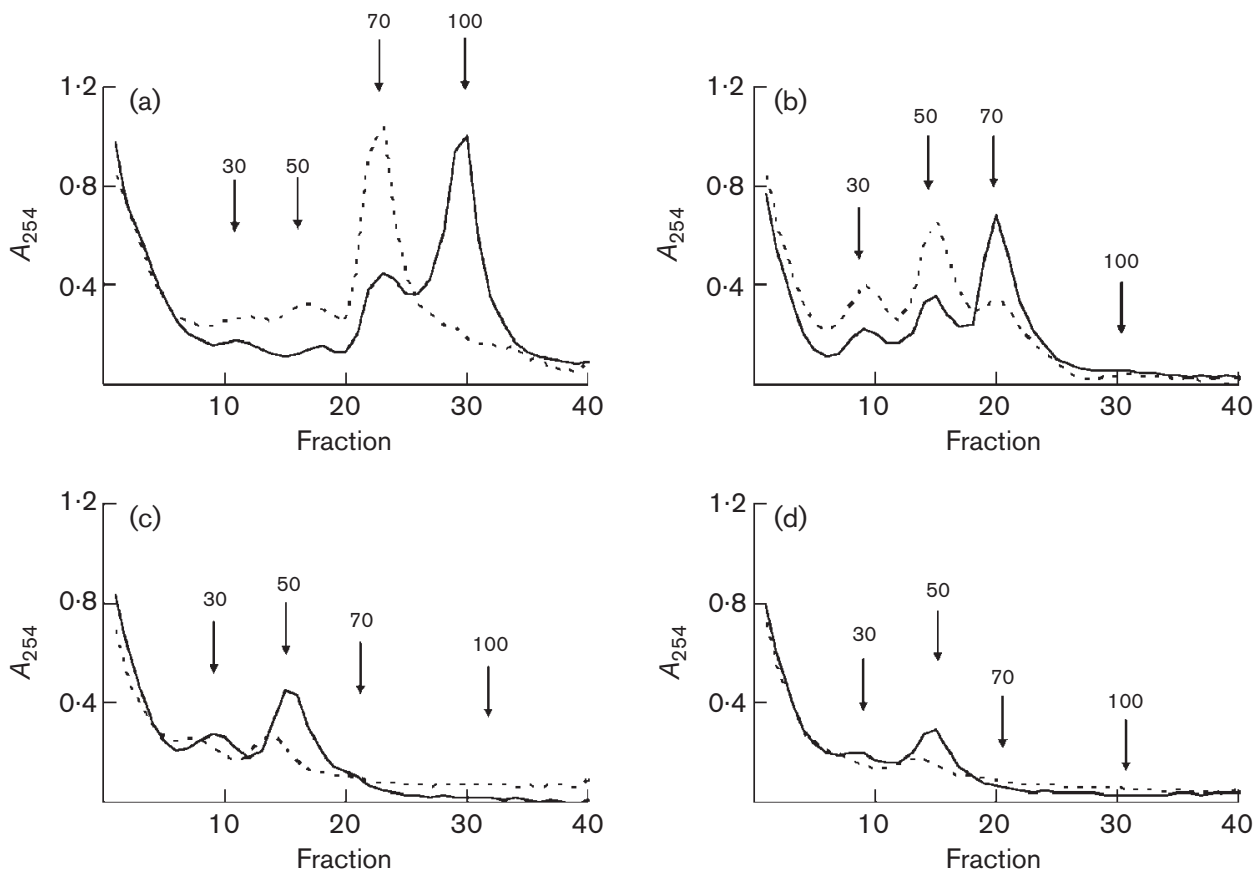

Fig. 2. Analyses of the ribosome content of cell-free extracts of $E$. coli W3110 (solid lines) and HMY15 (broken lines). Stationary-phase cultures were inoculated into fresh media acidified to $\mathrm{pH} 3$ using $\mathrm{HCl}$. Samples were taken for analysis before inoculation (a), immediately after inoculation (b) and after exposure to acid media for $3 \mathrm{~h}$ (c) and $5 \mathrm{~h}$ (d). Ribosome particles were separated by sucrose density-gradient centrifugation and identified by measuring the absorbance at $254 \mathrm{~nm}$ of gradient fractions. The relative positions of peaks corresponding to $30 \mathrm{~S}, 50 \mathrm{~S}, 70 \mathrm{~S}$ and $100 \mathrm{~S}$ particles are marked accordingly. The data presented are the results of a single experiment, but are typical of several replicate experiments. 


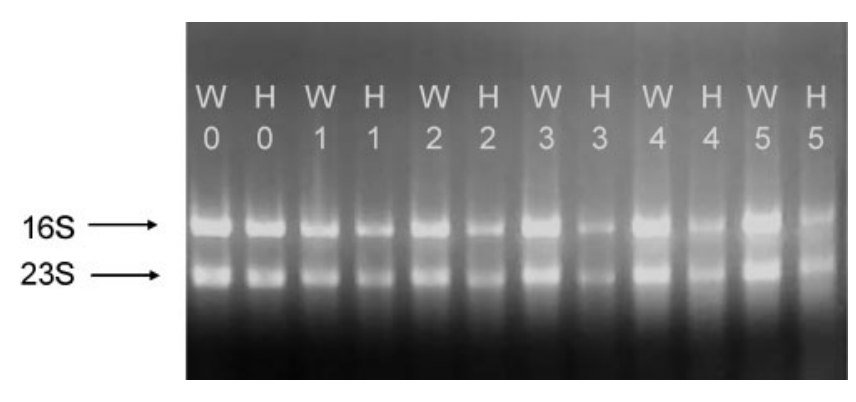

Fig. 3. Agarose gel analysis of rRNA extracted from stationaryphase cultures of $E$. coli after exposure to acid stress at $\mathrm{pH} 3$. The lanes are labelled $W$ for strain $W 3110$ and $H$ for strain HMY15. Numbers represent the exposure time in hours, with zero being control cultures before exposure. The positions of the $16 \mathrm{~S}$ and $32 \mathrm{~S}$ species, as identified by standard samples, are indicated. The data presented are the results of a single experiment, but are typical of several replicate experiments.

stress during stationary phase. Our previous studies of acid stress demonstrated that $r m f$ expression was higher during exponential-phase growth at low $\mathrm{pH}$ compared to unacidified media (El-Sharoud \& Niven, 2005). To determine if the $\mathrm{pH}$ of the growth medium influenced rmf expression during stationary phase, mutant strain HMY13, which carries a gene encoding an RMF- $\beta$-galactosidase hybrid protein, was grown at various $\mathrm{pH}$ levels and $r m f$ expression was estimated in early stationary phase by measuring $\beta$-galactosidase activity. As shown in Fig. 4, rmf expression levels were lower during stationary phase after growth in

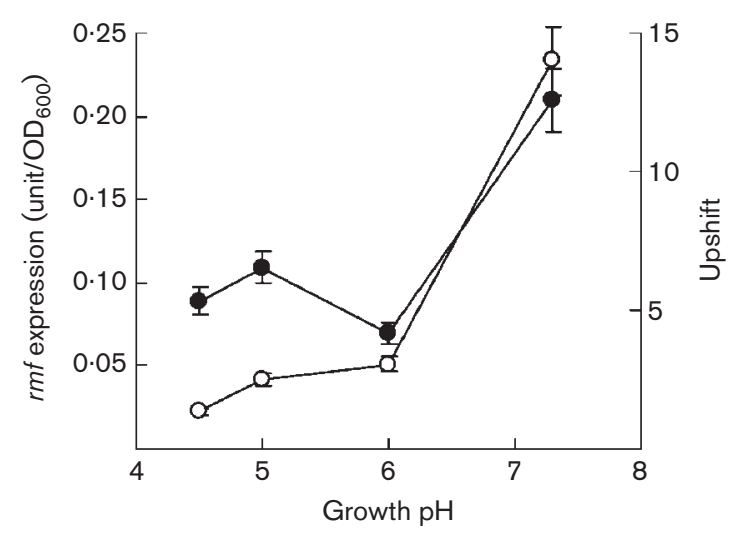

Fig. 4. Influence of growth $\mathrm{pH}$ on the expression of $r m f$ during stationary phase. Cultures of $E$. coli strain HMY13, which expresses an RMF- $\beta$-galactosidase fusion protein via the $r m f$ promoter, were grown to stationary phase at the stated $\mathrm{pH}$ and the expression of $r m f$ estimated from the $\beta$-galactosidase activity (๑). Upshift is the level of expression in stationary phase expressed as a factor of the level of expression in mid-exponential phase $(\bigcirc)$. Values are the means of three replicate determinations with a mean standard deviation of less than $9 \%$ of the mean, as represented by the error bars. acidified media than when cultures were grown in unacidified media. Compared to growth in unacidified medium, the net effect of growth in acidified medium was increased $r m f$ expression during exponential phase and decreased expression during stationary phase. The overall influence of acid conditions was therefore to reduce the upshift of $r m f$ expression typically associated with entry into stationary phase (Fig. 4).

The relative concentrations of ribosome particles in stationary-phase cultures of strains W3110 and HMY15 were compared after growth at various $\mathrm{pH}$ levels. It was observed that the degree of ribosome dimerization was lower in the parent strain after growth under acidic conditions (Fig. 5), as is consistent with lower rmf expression. This was accompanied by higher levels of $70 \mathrm{~S}$ and $50 \mathrm{~S}$ particles. No ribosome dimerization was observed in stationary-phase cultures following growth at $\mathrm{pH} 5.5$ or less. In the RMF-deficient mutant strain, growth under acidic conditions resulted in the detection of lower levels of $70 \mathrm{~S}$ ribosomes and increased levels of $50 \mathrm{~S}$ subunits in stationary phase. Although the analysis was not highly quantitative, there was no evidence that strains W3110 and HMY15 contained different total numbers of ribosomes, but

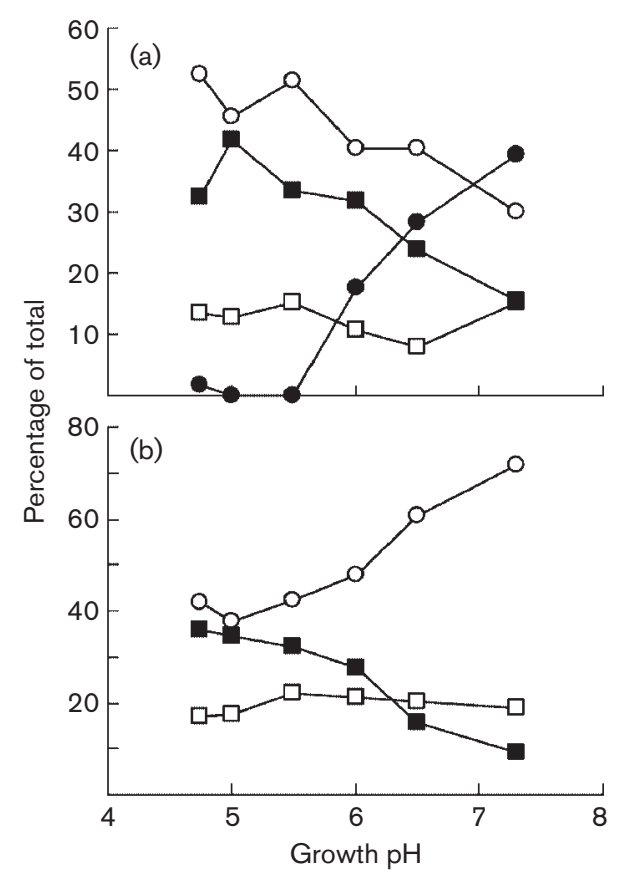

Fig. 5. Comparison of the relative proportions of ribosome particle types in cell-free extracts of stationary-phase cultures of E. coli W3110 (a) and HMY15 (b) following growth in media at different $\mathrm{pH}$ levels, acidified with $\mathrm{HCl}$. The proportions of 30S $(\square)$, 50S ( $\square), 70 S(\bigcirc)$ and 100S (0) particles were estimated following sucrose density-gradient centrifugation. The data presented are the means of duplicate determinations, the mean difference between determinations being $14 \%$ of the mean value. 
totals in both strains appeared to be reduced under acid conditions (data not shown).

\section{Influence of growth $\mathrm{pH}$ on resistance to acid challenge}

The data shown in Fig. 1 established that RMF had an influence on resistance to acid stress during stationary phase. It is known that previous exposure to acid conditions can confer increased resistance to subsequent acid stress (Rowbury, 1997; Foster, 1999). To determine if RMF plays any role in these processes, cultures of W3110 and HMY15 were grown to stationary phase at various $\mathrm{pH}$ levels before being exposed to acid $(\mathrm{HCl})$ challenge at $\mathrm{pH} 2.5$ for $1 \mathrm{~h}$. This degree of acid stress resulted in a reduction in viability of approximately $1.5 \mathrm{log}$ units in cultures of the parent strain grown in unacidified medium (Fig. 6). The degree of cell death was reduced to approximately $0.5 \log$ units in cultures grown at $\mathrm{pH} 4.75$, indicating that growth under acidic conditions had indeed resulted in increased resistance to subsequent acid stress in stationary phase.

The RMF-deficient mutant strain was shown to be more vulnerable to acid challenge than the parent strain when grown in unacidified medium, as was already demonstrated by the results in Fig. 1. However, the acid resistance of strain HMY15 increased markedly on growth under slightly acidic conditions, as the degree of cell death on acid challenge decreased from $5 \log$ units after growth at $\mathrm{pH} 7.3$ to $1.25 \mathrm{log}$ units after growth at $\mathrm{pH}$ 6.5. Between growth $\mathrm{pH}$ values of 6.5 and 5.5, the degree of cell death on acid challenge was similar to that of the parent strain. These data appear to suggest that RMF played no role in stationary-phase acquired acid tolerance since resistance to acid challenge increased on growth in acid $\mathrm{pH}$ with both strains. However, the acquired acid tolerance appeared to overcome the increased vulnerability to acid stress caused by RMF

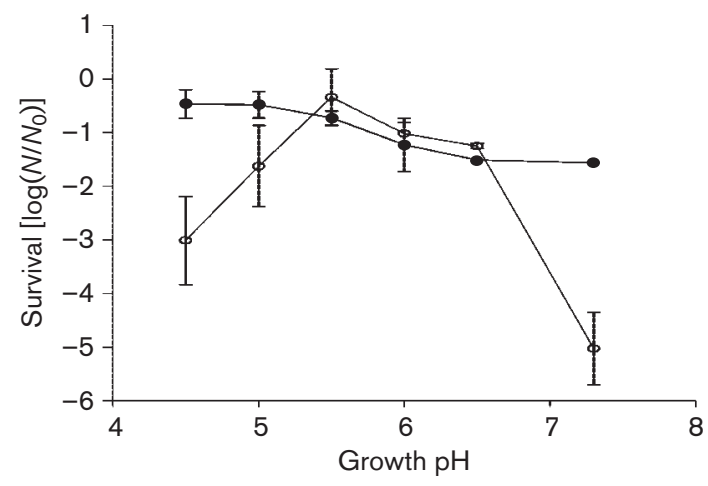

Fig. 6. Influence of growth medium $\mathrm{pH}$ on the survival of stationary-phase cultures of $E$. coli strain W3110 (O) and HMY15 (O) on exposure to $\mathrm{pH} 2.5$ for $1 \mathrm{~h}$. Values presented are the means of three separate experiments and the error bars represent \pm 1 SD. deficiency, as both strains showed similar resistance to acid challenge when grown under slightly acidic conditions. In cultures grown at less than $\mathrm{pH}$ 5.0, the mutant strain was again more vulnerable to acid challenge than the parent strain, indicating that RMF may play a role in acid resistance at lower growth $\mathrm{pH}$ values.

\section{DISCUSSION}

The data presented in this study demonstrated that stationary-phase cultures of an RMF-deficient mutant strain of E. coli (HMY15) were more vulnerable to acid stress at $\mathrm{pH} 3$ than cultures of the parent strain (W3110). This implied that RMF contributed to acid stress resistance in stationary phase, which complements previous observations that RMF enhances resistance to heat stress (Niven, 2004) and osmotic stress (Garay-Arroyo et al., 2000) during stationary phase. It therefore appears likely that RMF confers a generalized increased resistance to conditions that may result in lethal ribosome damage.

The nature of the protection conferred by RMF was investigated by monitoring the relative concentrations of ribosome particles in cell-free extracts of cultures after exposure to $\mathrm{pH} 3$. It was observed that acid challenge resulted in the breakdown of ribosomes into subunits and ultimately the loss of intact ribosomes over a period of $5 \mathrm{~h}$ in both the RMF-deficient mutant strain and the parent strain. This process was slower in the parent strain, and may therefore have been retarded by the presence of functional RMF. However, the parent strain retained a higher level of culture viability than the mutant after this treatment, despite suffering a similar degree of ribosome loss. Although RMF may thus confer a certain level of ribosome protection that retards ribosome dissociation, the degree of preservation of intact ribosome particles did not appear sufficient to fully explain the differences in acid sensitivity between strains HMY15 and W3110.

It is possible to consider that, following ribosome dissociation under acid stress, subsequent recovery and growth of cells when plated on unacidified medium is associated with the reconstitution of intact and functioning ribosomes. A similar situation was reported with Salmonella Typhimurium on recovery from sublethal heat stress (Genthner \& Martin, 1990). Despite the high degree of ribosome particle breakdown observed in both strains on acid stress, the rRNA of the parent strain remained relatively intact, even after exposure to acid medium for $5 \mathrm{~h}$. By contrast, rRNA degradation was clearly observed in the RMF-deficient mutant strain within the same period. It may therefore be the enhanced stability of the rRNA in the parent strain that was the significant factor in determining the higher level of acid resistance compared to the RMFdeficient mutant strain. This suggests that RMF may be involved in the preservation of the molecular elements from which ribosome subunits are composed, rather than in the preservation of intact ribosome particles. 
Our previous findings demonstrated that expression of the $r m f$ gene during exponential phase was higher when cultures were grown under acidic conditions (El-Sharoud \& Niven, 2005). This was consistent with gene expression being upregulated at low growth rates, mediated by cellular ppGpp concentrations, as reported by Izutsu et al. (2001). Expression was not therefore a function of the acidity of the medium per se, but of the resulting reduction in growth rate caused by the acid. It was observed that the peptide elongation rate was higher under acidic conditions in the parent strain than in the RMF-deficient strain (ElSharoud \& Niven, 2005). We therefore suggested that the function of RMF during exponential phase may be to preserve the efficiency of protein synthesis by inactivating and storing unrequired excess ribosomes during periods of low growth.

When E. coli is inoculated into unacidified media, rmf expression is low during exponential growth and increases substantially on entry into stationary phase (Yamagishi et al., 1993; Wada et al., 2000). In the current study, the expression of $r m f$ during stationary phase was observed to be lower after growth in acidified media than in unacidified media. The net result was that the upshift in rmf expression on the transition from exponential to stationary phase reported elsewhere was substantially reduced under acidic conditions. This observation is consistent with the hypothesis that the function of RMF is to inactivate unrequired ribosomes. A substantial increase in rmf expression is likely to be required on entry to stationary phase following a period of rapid growth since a large number of ribosomes will be transferring from an active to an inactive state. Conversely, following relatively slow growth in acidified medium, with fewer active ribosomes, correspondingly lower $r m f$ expression is required for their inactivation on entry to stationary phase.

The phenomenon by which the resistance of bacterial cells to acid stress is enhanced by prior exposure to acid conditions has been described extensively in the literature (Rowbury, 1997; Foster, 1999, 2000). The conclusion that RMF was important for survival during acid stress in stationary phase and the observation that $r m f$ expression was reduced after growth under acidic conditions invited further consideration of the role of RMF in this stress-adaptation mechanism. This subject is made complex by the existence of several overlapping mechanisms, and also by the varying stress conditions used by different researchers and varying terminology used to describe the phenomena observed. In the present study, the influence of RMF on $\mathrm{pH}$-dependent induced acid resistance in stationary-phase cultures (hereafter referred to as acid-tolerance response, ATR) was investigated. This was explored by growing cultures to stationary phase at various $\mathrm{pH}$ levels before subjecting them to acid challenge at $\mathrm{pH} 2.5$ for $1 \mathrm{~h}$. The RMF-deficient mutant strain was significantly more sensitive to acid challenge than the parent strain when grown in unacidified medium. However, the higher vulnerability of strain
HMY15 to acid challenge was completely overcome by growth at $\mathrm{pH}$ 5.5-6.5. This implied that ATR mechanisms were active in HMY15 and were therefore independent of RMF, and that ATRs were sufficient to overcome the increased vulnerability caused by the absence of RMF in the mutant strain.

Determination of the relative amounts of ribosome particles in stationary-phase cultures after growth at various $\mathrm{pH}$ levels resulted in the observation that the proportion of $100 \mathrm{~S}$ dimers decreased with growth $\mathrm{pH}$ and that none were detected at a growth $\mathrm{pH}$ of 5.5 or less. In that case, the differential sensitivity to acid between strains HMY15 and W3110 after growth at $\mathrm{pH} 5.5$ and less could be attributed to RMF, but not correlated with ribosome dimerization. This adds to a growing body of evidence suggesting that the function of RMF may not be directly dependent on the formation of $100 \mathrm{~S}$ dimers. In our previous study of RMF in relation to acid stress during exponential-phase growth, increased $r m f$ expression under acidic conditions did not result in the detection of increased ribosome dimerization (El-Sharoud \& Niven, 2005). Also, in studies of heat-stressed cells in stationary phase, $100 \mathrm{~S}$ dimers were demonstrated to be more sensitive to heat than 70S particles (Niven, 2004). Their formation could not therefore explain why RMF increased resistance to heat stress. It is possible that RMF works on two levels: firstly to inactivate and stabilize unrequired ribosomes in growing cells, and secondly to prevent rRNA degradation after ribosome dissociation. Clearly, the latter function is independent of ribosome dimerization. Despite a convincing body of evidence that dimers are associated with RMF, there is not yet any direct evidence demonstrating the formation of dimers in vivo. Further studies are required to elucidate the function and mechanism of action of RMF.

This study adds to our understanding of RMF function by demonstrating that RMF has a role in the resistance to acid stress in stationary-phase cultures of E. coli. This complements previous studies demonstrating similar roles in heatand osmotic-stressed cultures (Niven, 2004; Garay-Arroyo et al., 2000). This protein clearly plays an important part in the survival of $E$. coli and other bacteria expressing analogous genes (Hayashi et al., 2001; Yoshida et al., 2004) under harsh environments and has practical relevance to growth in fermented foods, pathogenicity and biotechnology. However, it generates intriguing questions about the role of ribosome dimerization in RMF function and highlights potentially differing functions in exponentialand stationary-phase cultures.

\section{ACKNOWLEDGEMENTS}

Walid M. El-Sharoud is grateful to the Government of Egypt for their financial support to carry out this study. The authors would also like to thank Dr Bernard Mackay for valuable discussions and Professor Akira Ishihama for his generous gift of the E. coli strains. 


\section{REFERENCES}

Apirakaramwong, A., Fukuchi, J.-I., Kashiwagi, K., Kakinuma, Y., Ito, E., Ishihama, A. \& Igarishi, K. (1998). Enhancement of cell death due to decrease in $\mathrm{Mg}^{2+}$ uptake by OmpC (cation-selective porin) deficiency in ribosome modulation factor-deficient mutant. Biochem Biophys Res Commun 251, 482-487.

El-Sharoud, W. M. \& Niven, G. W. (2005). The activity of ribosome modulation factor during growth of Escherichia coli under acidic conditions. Arch Microbiol 184, 18-24.

Foster, J. W. (1999). When protons attack: microbial strategies of acid adaptation. Curr Opin Microbiol 2, 170-174.

Foster, J. W. (2000). Microbial response to acid stress. In Bacterial Stress Responses, pp. 99-115. Edited by G. Storz \& R. Hengge-Aronis. Washington, DC: American Society for Microbiology.

Fukuchi, J., Kashiwagi, K., Yamagishi, M., Ishihama, A. \& Igarashi, K. (1995). Decrease in cell viability due to the accumulation of spermidine in spermidine acetyltransferase-deficient mutant of Escherichia coli. J Biol Chem 270, 18831-18835.

Garay-Arroyo, A., Colmenero-Flores, J. M., Garciarrubio, A. \& Covarrubias, A. A. (2000). Highly hydrophilic proteins in prokaryotes and eukaryotes are common during conditions of water deficit. J Biol Chem 25, 5668-5674.

Genthner, F. J. \& Martin, S. E. (1990). Ribosome assembly in Salmonella Typhimurium 7136 during recovery from heat shock. Food Microbiol 7, 53-67.

Hayashi, T., Makino, K., Ohnishi, M., Kurokawa, K., Ishii, K., Yokoyama, K., Han, C. G., Ohtsubo, E., Nakayama, K. \& other authors (2001). Complete genome sequence of enterohemorrhagic Escherichia coli $\mathrm{O} 157: \mathrm{H} 7$ and genomic comparison with a laboratory strain K-12. DNA Res 8, 11-22.

Ishihama, A. (1999). Modulation of the nucleoid, the transcription apparatus, and the translation machinery in bacteria for stationary phase survival. Genes Cells 4, 135-143.
Izutsu, K., Wada, A. \& Wada, C. (2001). Expression of ribosome modulation factor (RMF) in Escherichia coli requires ppGpp. Genes Cells 6, 665-676.

Kornblum, J. S., Projan, S. J., Moghazeh, S. L. \& Novick, R. P. (1988). A rapid method to quantitate non-labeled RNA species in bacterial cells. Gene 63, 75-85.

Maki, Y., Yoshida, H. \& Wada, A. (2000). Two proteins, YfiA and $\mathrm{YhbH}$, associated with resting ribosomes in stationary phase Escherichia coli. Genes Cells 5, 965-974.

Miller, J. H. (1972). Experiments in Molecular Genetics. Cold Spring Harbor Laboratory, NY: Cold Spring Harbor.

Niven, G. W. (2004). Ribosome modulation factor protects Escherichia coli during heat stress, but this may not be dependent on ribosome dimerisation. Arch Microbiol 162, 60-66.

Rowbury, R. J. (1997). Regulatory components, including integration host factor, CysB and H-NS, that influence $\mathrm{pH}$ responses in Escherichia coli. Lett Appl Microbiol 24, 319-328.

Wada, A. (1998). Growth phase coupled modulation of Escherichia coli ribosomes. Genes Cells 3, 203-208.

Wada, A., Yamazaki, Y., Fujita, N. \& Ishihama, A. (1990). Structure and probable genetic location of a "ribosome modulation factor" associated with $100 \mathrm{~S}$ ribosomes in stationary-phase Escherichia coli cells. Proc Natl Acad Sci U S A 87, 2657-2661.

Wada, A., Mikkola, R., Kurland, C. G. \& Ishihama, A. (2000). Growth phase-coupled changes of the ribosome profile in natural isolates and laboratory strains of Escherichia coli. J Bacteriol 182, 2893-2899.

Yamagishi, M., Matsushima, H., Wada, A., Sakagami, M., Fujita, N. \& Ishihama, A. (1993). Regulation of the Escherichia coli rmf gene encoding the ribosome modulation factor - growth phase-dependent and growth rate-dependent control. EMBO J 12, 625-630.

Yoshida, H., Yamamoto, H., Uchiumi, T. \& Wada, A. (2004). RMF inactivates ribosomes by covering the peptidyl transferase centre and entrance of peptide exit tunnel. Genes Cells 9, 271-278.

Edited by: C. Edwards 